\title{
Approximation of fixed points for Garcia-Falset mappings in a uniformly convex Banach space
}

\author{
Tanapat Chalarux ${ }^{\mathrm{a}}$, Khuanchanok Chaichana ${ }^{\mathrm{b}}$
}

a Department of Mathematics, Chiang Mai University, Chiang Mai 50200, Thailand.

${ }^{b}$ Advanced Research Center for Computational Simulation, Department of Mathematics, Chiang Mai University, Chiang Mai 50200, Thailand.

\begin{abstract}
The aim of this research is to introduce a novel iterative technique termed CC-iteration for identifying the fixed points of Garcia-Falset mappings. In uniformly convex Banach spaces, we establish both weak and strong convergence characteristics. Additionally, numerical examples of the iterative approach are presented in the form of a signal recovery application in a compressed sensing issue.
\end{abstract}

Keywords: Garcia-Falset mapping, Iteration, Convergence, Signal recovery. 2010 MSC: 37C25, 47H10, 54H25.

\section{Introduction}

Let $C$ be a nonempty subset of a Banach space $X$, Garcia-Falset et al. 3] presented a mapping $T: C \rightarrow X$ satisfying condition $\left(E_{\mu}\right)$ on $C$, that is, there exists $\mu \geq 1$ such that

$$
\|x-T y\| \leq \mu\|x-T x\|+\|x-y\|
$$

for all $x, y \in C$. It is noticeable that $T: C \rightarrow X$ satisfies condition $\left(E_{1}\right)$ if it is nonexpansive. By Lemma 7 in [15], $T: C \rightarrow C$ satisfies condition $\left(E_{3}\right)$ if it is a Suzuki mapping. In [3], they also determined the existence and asymptotic behavior of fixed points. Moreover, there are interesting studies on the fixed point problem for additional nonlinear mappings in [18, 19].

Many problems in various fields, such as image reconstruction [12, 14, 23] and signal processing [1, 13, 20, 21, 22, 24], can be modeled as fixed point problems. Numerous authors have presented various iterative

Email addresses: tanapatchalarux@gmail.com (Tanapat Chalarux), khuanchanok.c@cmu.ac.th (Khuanchanok Chaichana)

Received : May 01, 2021; Accepted: September 01, 2021; Online: September 03, 2021. 
approaches for fixed point numerical approximation. Ishikawa [5], Mann [6] and Noor [7] are some of the pioneers of iterative approaches for estimating fixed points in nonlinear mappings. In 2020, Usurelu et al. [17] showed the existence of fixed points for Garcia-Falset mappings in uniformly convex Banach spaces using the TTP-iteration introduced in [16]. A number of convergence results have also been obtained using this iterative strategy. This effort will provide a new iterative method in advance of inspiring research. As follows is the definition of the CC-iteration method: $x_{0} \in C$ and

$$
\begin{aligned}
z_{n} & =\left(1-e_{n}\right) x_{n}+e_{n} T x_{n}, \\
y_{n} & =\left(1-c_{n}-d_{n}\right) z_{n}+c_{n} T x_{n}+d_{n} T z_{n}, \\
x_{n+1} & =\left(1-a_{n}-b_{n}\right) T x_{n}+a_{n} T y_{n}+b_{n} T z_{n},
\end{aligned}
$$

for all $n \geq 0$, where $\left\{a_{n}\right\},\left\{b_{n}\right\},\left\{c_{n}\right\},\left\{d_{n}\right\},\left\{e_{n}\right\},\left\{a_{n}+b_{n}\right\}$ and $\left\{c_{n}+d_{n}\right\}$ are sequences in $(0,1)$. Using this iterative approach, we derive both weak and strong convergence theorems for Garcia-Falset mappings in uniformly convex Banach spaces, as well as a conclusion pertaining to the presence of fixed points for these mappings. All known results supporting the proving of key theorems will be detailed in the next section. Finally, the application of compressed sensing signal reconstruction will be studied in the last section, and the results will be compared to those of Noor [7] and Thakur et al. [16].

\section{Main Results}

We assume that $C$ is a nonempty closed convex subset of a real Banach space $X$ and $T: C \rightarrow C$ is a Garcia-Falset mapping for the rest of this section. Recall that if a fixed point set $\{z \in C \mid T z=z\}$ is nonempty and for every fixed point $z \in C$ and for each $x \in C$,

$$
\|z-T x\| \leq\|z-x\|
$$

then a mapping $T: C \rightarrow C$ is said to be quasi-nonexpansive.

Lemma 2.1. Let the fixed point set of $T$ is nonempty and let $\left\{x_{n}\right\}$ be a sequence defined by the iteration (1) where $x_{0} \in C$. Then $\lim _{n \rightarrow \infty}\left\|x_{n}-p\right\|$ exists for any fixed point $p$.

Proof. Let $p$ be a fixed point of $T$. Since the mapping $T$ is quasi-nonexpansive by [3, Proposition 1], we have

$$
\left\|T z_{n}-p\right\| \leq\left\|z_{n}-p\right\| \leq\left(1-e_{n}\right)\left\|x_{n}-p\right\|+e_{n}\left\|T x_{n}-p\right\| \leq\left\|x_{n}-p\right\| .
$$

By (2), we obtain

$$
\begin{aligned}
\left\|T y_{n}-p\right\| \leq\left\|y_{n}-p\right\| & \leq\left(1-c_{n}-d_{n}\right)\left\|z_{n}-p\right\|+c_{n}\left\|T x_{n}-p\right\|+d_{n}\left\|T z_{n}-p\right\| \\
& \leq\left(1-c_{n}-d_{n}\right)\left\|x_{n}-p\right\|+c_{n}\left\|x_{n}-p\right\|+d_{n}\left\|x_{n}-p\right\| \\
& =\left\|x_{n}-p\right\| .
\end{aligned}
$$

By (2) and (3), we have

$$
\begin{aligned}
\left\|x_{n+1}-p\right\| & \leq\left(1-a_{n}-b_{n}\right)\left\|T x_{n}-p\right\|+a_{n}\left\|T y_{n}-p\right\|+b_{n}\left\|T z_{n}-p\right\| \\
& \leq\left(1-a_{n}-b_{n}\right)\left\|x_{n}-p\right\|+a_{n}\left\|x_{n}-p\right\|+b_{n}\left\|x_{n}-p\right\| \\
& =\left\|x_{n}-p\right\| .
\end{aligned}
$$

It is noticeable that $\left\{\left\|x_{n}-p\right\|\right\}$ is bounded and nonincreasing for each fixed point $p$, that is, $\lim _{n \rightarrow \infty}\left\|x_{n}-p\right\|$ exists.

The proof of the main theorem can be supported by the following Schu's lemma. 
Lemma 2.2. [9] Let $X$ be a uniformly convex Banach space and let $\left\{\delta_{n}\right\}$ be a sequence such that $0<\bar{\delta} \leq$ $\delta_{n} \leq \delta^{*}<1$ for all $n \geq 1$ and for some positive real numbers $\bar{\delta}, \delta^{*}$. If sequences $\left\{a_{n}\right\}$ and $\left\{b_{n}\right\}$ in $X$ are such that $\limsup _{n \rightarrow \infty}\left\|a_{n}\right\| \leq q$, $\limsup _{n \rightarrow \infty}\left\|b_{n}\right\| \leq q$ and $\lim _{n \rightarrow \infty}\left\|\delta_{n} a_{n}+\left(1-\delta_{n}\right) b_{n}\right\|=q$ for some $q \geq 0$, then $\lim _{n \rightarrow \infty}\left\|a_{n}-b_{n}\right\| \stackrel{n \rightarrow \infty}{=} 0$.

Theorem 2.3. Let $X$ be uniformly convex and let $\left\{x_{n}\right\}$ be a sequence defined by the iteration (1), where $x_{0} \in C$ and $\left\{d_{n}\right\}$ is bounded away from 0 and 1 for all $n \geq 0$. The fixed point set of $T$ is nonempty if and only if $\left\{x_{n}\right\}$ is bounded and $\lim _{n \rightarrow \infty}\left\|T x_{n}-x_{n}\right\|=0$.

Proof. Suppose that the fixed point set of $T$ is nonempty and let $p$ be a fixed point of $T$. By Lemma 2.1. there exists $r \geq 0$ such that $r=\lim _{n \rightarrow \infty}\left\|x_{n}-p\right\|$ and the sequence $\left\{x_{n}\right\}$ is bounded. Next, we will show that $\lim _{n \rightarrow \infty}\left\|T x_{n}-x_{n}\right\|=0$. Taking limsup in 2, we obtain

$$
\limsup _{n \rightarrow \infty}\left\|z_{n}-p\right\| \leq \limsup _{n \rightarrow \infty}\left\|x_{n}-p\right\|=r .
$$

By the quasinonexpansiveness of $T$, we have

$$
\limsup _{n \rightarrow \infty}\left\|T x_{n}-p\right\| \leq \limsup _{n \rightarrow \infty}\left\|x_{n}-p\right\|=r .
$$

On the others hand,

$$
\begin{aligned}
\left\|x_{n+1}-p\right\| & \leq\left(1-a_{n}-b_{n}\right)\left\|T x_{n}-p\right\|+a_{n}\left\|T y_{n}-p\right\|+b_{n}\left\|T z_{n}-p\right\| \\
& \leq\left(1-b_{n}\right)\left\|x_{n}-p\right\|+b_{n}\left\|z_{n}-p\right\| .
\end{aligned}
$$

Therefore,

$$
\left\|x_{n+1}-p\right\|-\left\|x_{n}-p\right\| \leq b_{n}\left(\left\|x_{n+1}-p\right\|-\left\|x_{n}-p\right\|\right) \leq\left\|z_{n}-p\right\|-\left\|x_{n}-p\right\|,
$$

that is,

$$
\left\|x_{n+1}-p\right\| \leq\left\|z_{n}-p\right\|
$$

Taking lim inf in the above inequality, we get

$$
r \leq \liminf _{n \rightarrow \infty}\left\|x_{n+1}-p\right\| \leq \liminf _{n \rightarrow \infty}\left\|z_{n}-p\right\| \leq \limsup _{n \rightarrow \infty}\left\|z_{n}-p\right\| \leq r .
$$

Hence,

$$
\lim _{n \rightarrow \infty}\left\|\left(1-e_{n}\right)\left(x_{n}-p\right)+e_{n}\left(T x_{n}-p\right)\right\|=\lim _{n \rightarrow \infty}\left\|z_{n}-p\right\|=r .
$$

Combining (5)-(7) and Lemma 2.2, we can conclude that $\lim _{n \rightarrow \infty}\left\|T x_{n}-x_{n}\right\|=0$. Conversely, suppose that the sequence $\left\{x_{n}\right\}$ is bounded and $\lim _{n \rightarrow \infty}\left\|T x_{n}-x_{n}\right\|=0$. Next, suppose that $p \in A\left(C,\left\{x_{n}\right\}\right)$. Since $T$ satisfies condition $(E)$, the following relation is obtained:

$$
\begin{aligned}
r\left(T p,\left\{x_{n}\right\}\right) & =\limsup _{n \rightarrow \infty}\left\|x_{n}-T p\right\| \\
& \leq \limsup _{n \rightarrow \infty}\left(\mu\left\|T x_{n}-x_{n}\right\|+\left\|x_{n}-p\right\|\right) \\
& \leq \limsup _{n \rightarrow \infty}\left\|x_{n}-p\right\| \\
& =r\left(p,\left\{x_{n}\right\}\right) .
\end{aligned}
$$

Therefore, $T p \in A\left(C,\left\{x_{n}\right\}\right)$. By the uniqueness of asymptotic centers, we have $p=T p$, that is, the fixed point set of $T$ is nonempty. 
Suantai determined the following lemma, which we will use to establish the next outcome.

Lemma 2.4. [11] Let $X$ be a Banach space that satisfies Opial's property and let $\left\{a_{n}\right\}$ be a sequence in $X$. Let $a, b$ in $X$ be such that $\lim _{n \rightarrow \infty}\left\|a_{n}-a\right\|$ and $\lim _{n \rightarrow \infty}\left\|a_{n}-b\right\|$ exist. If $\left\{a_{n_{i}}\right\}$ and $\left\{a_{m_{i}}\right\}$ are subsequences of $\left\{a_{n}\right\}$ that weakly converge to $a$ and $b$, respectively, then $a=b$.

Theorem 2.5. Let $X$ be uniformly convex with Opial's property. Let $T$ and $\left\{x_{n}\right\}$ be the same in Theorem 2.3 and the fixed poined set of $T$ is nonempty. Then $\left\{x_{n}\right\}$ weakly converges to a fixed point of $T$.

Proof. We have $\left\{x_{n}\right\}$ is bounded sequence, $\lim _{n \rightarrow \infty}\left\|x_{n}-p\right\|$ exists for all fixed point $p$ and $\lim _{n \rightarrow \infty}\left\|x_{n}-T x_{n}\right\|=0$ by Lemma 2.1 and Theorem 2.3. Let $\left\{x_{n_{i}}\right\}$ and $\left\{x_{m_{i}}\right\}$ be subsequences of $\left\{x_{n}\right\}$ weakly converging to $z_{1}$ and $z_{2}$, respectively. Then $\lim _{i \rightarrow \infty}\left\|x_{n_{i}}-T x_{n_{i}}\right\|=\lim _{i \rightarrow \infty}\left\|x_{m_{i}}-T x_{m_{i}}\right\|=0$. We obtain $z_{1}, z_{2} \in C$ since $C$ is closed and convex, also by Mazur's theorem. As the demiclosedness at zero of $I-T$ from [3, Theorem 1], we have $z_{1}, z_{2}$ are fixed points. By Lemma 2.4, we can conclude that $z_{1}=z_{2}$. Therefore, $\left\{x_{n}\right\}$ weakly converges to a fixed point of $T$.

The next two results present strong convergence for Garcia-Falset mappings.

Theorem 2.6. Let $C$ be a nonempty, compact and convex subset of a uniformly convex Banach space $X$. Let $T$ and $\left\{x_{n}\right\}$ be as same as in Theorem 2.3. If the fixed point set of $T$ is nonempty, then $\left\{x_{n}\right\}$ strongly converges to a fixed point of $T$.

Proof. This proof is the same as the proof of [17, Theorem 3.4].

Theorem 2.7. Let $C$ be a nonempty, closed and convex subset of a uniformly convex Banach space $X$. Let $T$ and $\left\{x_{n}\right\}$ be as same as in Theorem [2.3. If $T$ satisfies the condition $(I)$ in [10] and the fixed point set of $T$ is nonempty, then $\left\{x_{n}\right\}$ strongly converges to a fixed point of $T$.

Proof. The proof is the same as the proof of [17, Theorem 3.5].

\section{Applications}

We use our iterative method to solve the issue of retrieving the original signal from compressive measurements in this section. Let $\bar{x} \in \mathbb{R}^{N}$ and $y \in \mathbb{R}^{M}$ be the original signal and the observed data, respectively. Consider

$$
y=A \bar{x}+\varepsilon,
$$

where $A \in \mathbb{R}^{M \times N}(M<N)$ and $\varepsilon \in \mathbb{R}^{M}$ represents the Gaussian noise with $N\left(0, \sigma^{2}\right)$. The compressive sensing signal reconstruction described in the preceding equation is what we want to solve. However, it is well known that solving (8) is identical to the LASSO problem:

$$
\min _{x \in \mathbb{R}^{N}} \frac{1}{2}\|A x-y\|_{2}^{2} \text { subject to }\|x\|_{1} \leq \zeta
$$

where $\zeta>0$. 9 can be seen as the fixed point problem through the following settings:

$$
T=P_{D}\left(I-\frac{1}{\|A\|^{2}} \nabla g\right), \text { where } g(x)=\frac{1}{2}\|A x-y\|_{2}^{2} \text { and } D=\left\{x \in \mathbb{R}^{N}:\|x\|_{1} \leq \zeta\right\} .
$$

We have known that $(I-\kappa \nabla g)$ is nonexpansive for any $0<\kappa<\frac{2}{\|A\|^{2}}$ (see [4]). In addition, $P_{D}$ has closed forms which is the projection onto the closed $l_{1}$ ball in $\mathbb{R}^{N}$ (see [2]). Then, for (9), we provide a numerical 
solution. We look at how the CC-iteration (1) behaves and compare it to two other iterative methods: the Noor iteration [7] and the TTP-iteration [16]. The Noor iteration was defined as follows: $x_{0} \in C$ and

$$
\begin{aligned}
z_{n} & =\left(1-\gamma_{n}\right) x_{n}+\gamma_{n} T x_{n}, \\
y_{n} & =\left(1-\beta_{n}\right) x_{n}+\beta_{n} T z_{n}, \\
x_{n+1} & =\left(1-\alpha_{n}\right) x_{n}+\alpha_{n} T y_{n},
\end{aligned}
$$

for all $n \geq 0$, where $\left\{\alpha_{n}\right\},\left\{\beta_{n}\right\},\left\{\gamma_{n}\right\}$ are sequences of real numbers in $(0,1)$, and the TTP-iteration was defined as follows: $x_{0} \in C$ and

$$
\begin{aligned}
z_{n} & =\left(1-\gamma_{n}\right) x_{n}+\gamma_{n} T x_{n}, \\
y_{n} & =\left(1-\beta_{n}\right) z_{n}+\beta_{n} T z_{n}, \\
x_{n+1} & =\left(1-\alpha_{n}\right) T z_{n}+\alpha_{n} T y_{n},
\end{aligned}
$$

for all $n \geq 0$, where $\left\{\alpha_{n}\right\},\left\{\beta_{n}\right\},\left\{\gamma_{n}\right\}$ are sequences of real numbers in $(0,1)$. Let $N=2^{12}$ and $M=2^{11}$ be the size of signal. Suppose that there are $m$ nonzero elements in the original signal, then generate the Gaussian matrix $A$ by using $\operatorname{randn}(M, N), \sigma=0.1$ and $\zeta=m$. Choose $x_{0}=A^{t} y$ as the initial point. For any $n \geq 0$, let $\alpha_{n}=\frac{3 n+3}{4 n+12}, \beta_{n}=c_{n}=\frac{\sqrt{15 n+10}-(3 n+3)^{\frac{1}{4}}}{10 \sqrt{15 n+10}}, \gamma_{n}=e_{n}=\sqrt{\frac{n+1}{16 n+15}}, a_{n}=\frac{4 n+4}{5 n+15}, b_{n}=\frac{n+1}{10 n+30}$ and $d_{n}=\frac{4\left(\sqrt{15 n+10}-(3 n+3)^{\frac{1}{4}}\right)}{5 \sqrt{15 n+10}}$. Then, we compare the accuracy between the recovered signals with the meansquared error: $\operatorname{MSE}_{n}=\frac{1}{N}\left\|x_{n}-\bar{x}\right\|^{2}<5 \times 10^{-5}$.

\begin{tabular}{ccccc}
\hline \multirow{2}{*}{ Iterative schemes } & & \multicolumn{3}{c}{$m$ Nonzero Elements } \\
\cline { 3 - 5 } & & $m=25$ & $m=50$ & $m=100$ \\
\hline \multirow{2}{*}{ Noor } & Elapsed Time (s) & 0.1346 & 0.2339 & 1.4834 \\
\cline { 2 - 5 } & No. of Iter. & 85 & 171 & 949 \\
\hline \multirow{2}{*}{ TTP } & Elapsed Time (s) & 0.0815 & 0.1423 & 1.0501 \\
\cline { 2 - 5 } & No. of Iter. & 49 & 101 & 593 \\
\hline \multirow{2}{*}{ CC } & Elapsed Time (s) & 0.0594 & 0.0951 & 0.5642 \\
\cline { 2 - 5 } & No. of Iter. & 38 & 76 & 426 \\
\hline
\end{tabular}

Table 1: Three iterative methods are numerically compared.

In Table 1, different numbers of nonzero elements were used in the numerical experiments: $m=25,50$ and 100. For each iterative method in these three situations, the elapsed periods and number of iterations are recorded. The CC-iteration uses less time on average than the other two iterative methods. Likewise, the CC-iteration's number is lower than the others. We also show the recovery signals for $m=100$ in Figure 1. In these specific instances, iteration enhances the numerical results. We compute the errors of each reconstructed signal in Figure 2 to detect the differences between these outcomes. 

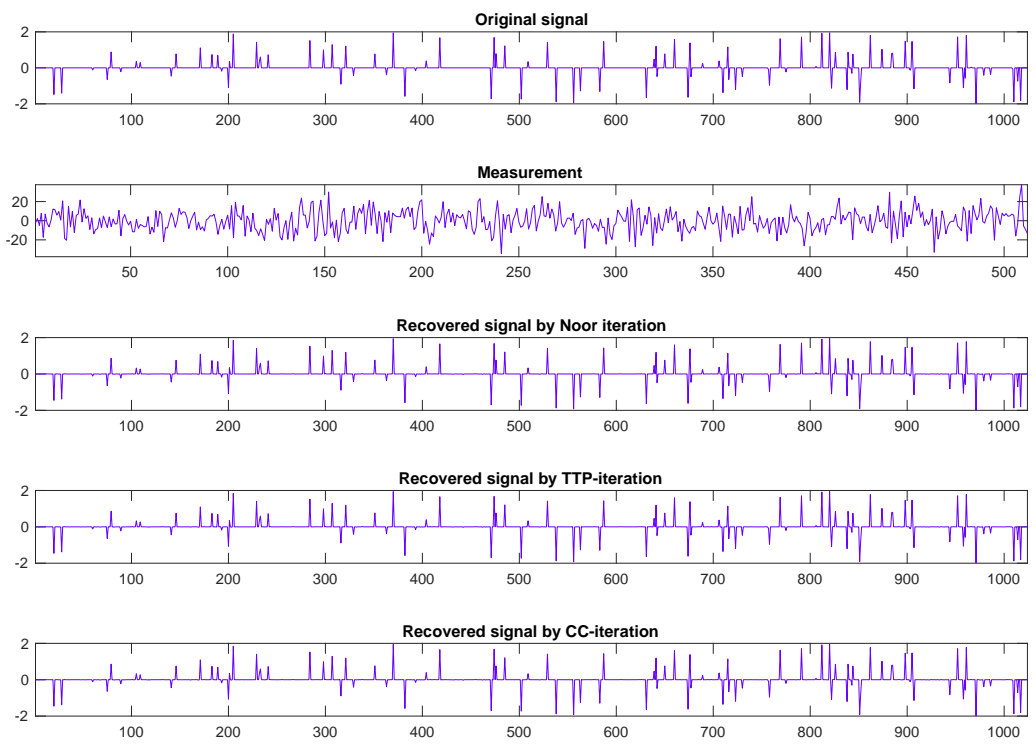

Figure 1: From top to bottom: the original signal, the measurement, and the recovery signals by the Noor iteration, the TTPiteration and CC-iteration, respectively when $m=100$.

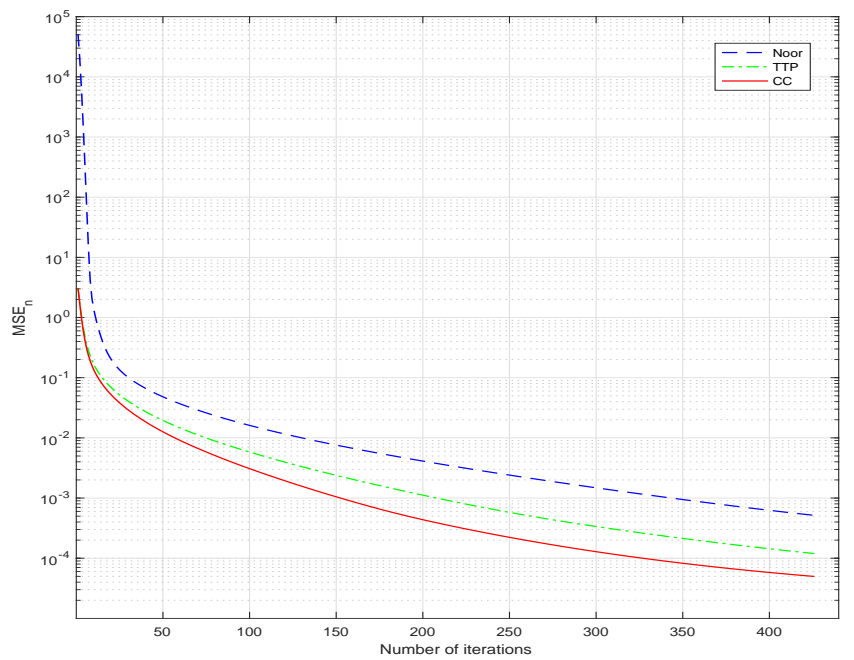

Figure 2: Mean-squared error versus number of iterations when $m=100$.

\section{Conclusion}

In conclusion, we solve the fixed points of Garcia-Falset mappings using an up-to-date iterative technique. Furthermore, under specific situations, we confirm the iterative scheme's weak and strong convergence findings. The iterative technique was then applied to the problem of signal recovery in compressed sensing. When compared to other iterative systems, the numerical studies of our iterative method yield better results.

\section{Acknowledgement}

This research was supported by CMU Junior Research Fellowship Program. 


\section{References}

[1] P. L. Combettes, V. R. Wajs, Signal recovery by proximal forward-backward splitting, Multiscale Model. Simul. 4 (2005) 1168-1200.

[2] J. Duchi, S. Shalev-Shwartz, Y. Singer, T. Chandra, Efficient projections onto the $l_{1}$-ball for learning in high dimensions, In: Proceedings of the 25th International Conference on Machine Learning, Helsinki, Finland. (2008) 272-279.

[3] J. García-Falset, E. Llorens-Fuster, T. Suzuki, Fixed point theory for a class of generalized nonexpansive mappings, J. Math. Anal. Appl. 375 (2011) 185-195.

[4] H. Iiduka, W. Takahashi, Strong convergence theorems for nonexpansive nonself-mappings and inverse-strongly-monotone mappings, J. Convex Anal. 11 (2004) 69-79.

[5] S. Ishikawa, Fixed points by a new iteration method, Proc. Amer. Math. Soc. 4(1) (1974) 147-150.

[6] W. R. Mann, Mean value methods in iteration, Proc. Amer. Math. Soc. 4 (1953) 506-510.

[7] M. A. Noor, New approximation schemes for general variational inequalities, J. Math. Anal. Appl. 251(1) (2000) 217-229.

[8] Z. Opial, Weak convergence of the sequence of successive approximations for nonexpansive mappings, Bull. Amer. Math. Soc. 73(4) (1967) 591-598.

[9] J. Schu, Weak and strong convergence to fixed points of asymptotically nonexpansive mappings, Bull. Aust. Math. Soc. 43(1) (1991) 153-159.

[10] H. F. Senter, W. G. Dotson, Approximating fixed points of nonexpansive mappings, Proc. Amer. Math. Soc. 44 (1974) 375-380.

[11] S. Suantai, Weak and strong convergence criteria of Noor iterations for asymptotically nonexpansive mappings, J. Math. Anal. Appl. 311(2) (2005) 506-517.

[12] R. Suparatulatorn, P. Charoensawan, K. Poochinapan, Inertial self-adaptive algorithm for solving split feasible problems with applications to image restoration, Math. Methods Appl. Sci. 42(18) (2019) 7268-7284.

[13] R. Suparatulatorn, A. Khemphet, Tseng type methods for inclusion and fixed point problems with applications, Mathematics. 7(12) (2019) 1175.

[14] R. Suparatulatorn, A. Khemphet, P. Charoensawan, S. Suantai, N. Phudolsitthiphat, Generalized self-adaptive algorithm for solving split common fixed point problem and its application to image restoration problem, Int. J. Comput. Math. 97(7) (2020) 1431-1443.

[15] T. Suzuki, Fixed point theorems and convergence theorems for some generalized nonexpansive mappings, J. Math. Anal. Appl. 340(2) (2008) 1088-1095.

[16] B. S. Thakur, D. Thakur, M. Postolache, A new iteration scheme for approximating fixed points of nonexpansive mappings, Filomat. 30(10) (2016) 2711-2720.

[17] G. I. Usurelu, A. Bejenaru, M. Postolache, Operators with property (E) as concerns numerical analysis and visualization, Numer. Funct. Anal. Optim. 41(11) (2020) 1398-1419.

[18] P. Cholamjiak, W. Cholamjiak, Fixed point theorems for hybrid multivalued mappings in Hilbert spaces, J. Fixed Point Theory Appl. 18 (2016) 673-688.

[19] P. Cholamjiak, A. A. Abdou, Y. J. Cho, Proximal point algorithms involving fixed points of nonexpansive mappings in CAT(0) spaces, Fixed Point Theory Appl. 2015 (2015) 227.

[20] S. Kesornprom, N. Pholasa, P. Cholamjiak, On the convergence analysis of the gradient-CQ algorithms for the split feasibility problem, Numerical Algorithms. 84(3) (2020) 997-1017.

[21] S. Kesornprom, P. Cholamjiak, Proximal type algorithms involving linesearch and inertial technique for split variational inclusion problem in hilbert spaces with applications, Optimization. 68(12) (2019) 2369-2395.

[22] S. Suantai, S. Kesornprom, P. Cholamjiak, A new hybrid CQ algorithm for the split feasibility problem in Hilbert spaces and its applications to compressed sensing, Mathematics. 7(9) (2019) 789.

[23] R. Suparatulatorn, P. Charoensawan, K. Poochinapan, S. Dangskul, An algorithm for the split feasible problem and image restoration, RACSAM. Rev. R. Acad. Cienc. Exactas Fis. Nat. Ser. A Mat. 115 (2021). https://doi.org/10.1007/s13398020-00942-z.

[24] R. Suparatulatorn, P. Charoensawan, A. Khemphet, An inertial subgradient extragradient method of variational inequality problems involving quasi-nonexpansive operators with applications, Math. Methods Appl. Sci. (2021). https://doi.org/10.1002/mma.7576. 